\title{
Über eine Analogie zwischen rotierender Scheibe und belasteter Kreisplatte.
}

\author{
Von L. FőPPL in Dresden.
}

I den folgenden Zeilen wird der Spannungs- und Formänderungszustand einer gleichmäBig rotierenden Scheibe (unter 1) nach der üblichen Darstellung behandelt und im Anschluß daran (unter 2) dasselbe für die symmetrisch belastete Kreisplatte ausgefiihrt, in beiden Fällen bei veränderlicher Dicke. Die vollkommene Analogie beider Aufgaben hinsichtlioh der Spannung wie der Zerrung tritt dabei deutlich hervor, so daB Lösungen der einen nnmittelbar auch Lösungen für die andere ergeben; hiervon wird an einem Beispiel Gebrauch gemacht, indem die bekannte Lösung für rotierende Scheiben mit veranderlicher Dicke auf die bisher nubekannte Lösung für symmetrisch belastete Kreisplatten von veränderlicher Stärke ïbertragen wird. Der Hauptvorteil dieser neuen Ana. logie dürfte neben der Oekonomie der Denkarbeit, die sie gestattet, in einer Oekonomie der Versuche zu erblicken sein, da sich auch die Versuchsergebnisse von dem einen aui den anderen Fall übertragen lassen. Als Grundannahmen für die Berechnung der Platte dienen die üblichen, gewöhnlich nach Kirchhoff bezeichneten, wonach nur die Biegung der Platte berücksichtigt wird.

1. Rotierende Scheibe mit veränderlicher Dicke. Wir denken uns eine kreissymmetrische Scheibe mit veränderlicher Dicke $h$, die mit konstanter Winkelgeschwindigkeit $\omega$ rotiert. Es bildet sich dabei ein achsensymmetrischer Spannungs- und Formänderungszustand aus. In erster Annäherung nimmt man gleichmäßige Verteilung der Spannungen über die Dicke der Platte an jeder Stelle an ${ }^{1}$ ), so daB

$$
h \sigma_{r}=T_{r} \text { und } h \sigma_{t}=T_{t}
$$

die Spannungsresultanten für radiale und tangentiale Schnitte senkrecht znr Mittelfläche, bezogen auf die Längeneinheit in der Mittelfläche, bedeuten. Die Gleichgewichtsbedingung für ein in dieser Weise aus der Platte ausgeschnittenes Element lautet dann bekanntlich:

$$
\frac{d T_{r}}{d r}=\frac{T_{t}-T_{r}}{r}-\mu \omega^{2} r h
$$

Diese rein statische Bedingung liefert uns nur die eine Gleichung für die beiden Unbekannten $T_{r}$ und $T_{t}$.

Um den Formänderungszustand zu beschreiben, bezeichnen wirmit $\varrho$ den Zuwachs, den der Radius $r$ der Scheibenmitteifläche infolge der Formänderung erfährt, so daB die auf die Längeneinheit bezogenen Dehnungen der Mittelflăche in Richtung des Radius und senkrecht dazu dureh

$$
\varepsilon_{r}=\frac{d \varrho}{d r}, \quad \varepsilon_{t}=\frac{\varrho}{r} .
$$

ausgedrückt werden. Nach dem Hookeschen Gesetz bestehen zwischen den Formänderungs- und Spannungsgrößen die Beziehungen:

$$
\frac{d \varrho}{d r}=\frac{1}{E h}\left(T_{r}-\frac{1}{m} T_{t}\right), \quad \stackrel{\varrho}{r}=\frac{1}{E h}\left(T_{t}-\frac{1}{m} T_{r}\right)
$$

oder nach den Spannungsresultanten aufgelöst:

$$
T_{r}=\frac{m^{2}}{m^{2}-1} E h\left(\frac{d \underline{Q}}{d r}+\frac{1}{m} \frac{\varrho}{r}\right), \quad T_{t}=\frac{m^{2}}{m^{2}-1} E h\left(\frac{g}{r}+\frac{1}{m} \frac{d}{d} \frac{g}{r}\right) \quad . \quad .
$$

Nach Einsetzen dieser Werte für $T_{r}$ und $T_{t}$ in die obige Gl. (1) erhält man unter Berüicksichtigung der Veränderlichkeit von $h$;

$$
\frac{d^{2} \varrho}{d r^{2}}+\frac{1}{r} \frac{d \varrho}{d r}-\frac{\varrho}{r^{2}}+\frac{1}{h} \frac{d h}{d r}\left(\begin{array}{l}
d r \\
d r
\end{array} \frac{1}{m} \stackrel{\varrho}{r}\right)=-\mu \omega^{2} \frac{m^{2}-1}{m^{2} E} r
$$

Damit ist die Aufgabe auf diese Differentialgleichung für $\varrho$ zurückgeführt, deren Lösung sich leicht angeben läßt, wenn $h$ in seiner Abhängigkeit von $r$ durch ein Potenzgesetz $h=c r^{n}$ gegeben ist. 
Häufig geht man auch so vor ${ }^{1}$ ), daß man aus den Gl. (3) $\varrho$ eliminiert. Dann ergibt sich:

$$
\frac{1}{h}\left(T_{r}-\frac{1}{m} T_{t}\right)=\frac{d}{d r}\left[\frac{r}{h}\left(T_{t}-\frac{1}{m} T_{r}\right)\right] \text {. . . . . . . . }
$$

Diese Gleichung bestimmt zusammen mit Gl. (1) $T_{r}$ und $T_{t}$. Durch Einführung einer Art Spannungsfunktion $F$, die mit den Spannungsresultanten durch

$$
T_{r}=\frac{F}{r}, \quad T_{t}=\frac{d F}{d r}+\mu \omega^{2} h r^{2} \quad . \quad . \quad . \quad . \quad . \quad . \quad .
$$

verbunden ist, wird Gl. (1) identisch befriedigt, während Gl. (5) als Bestimmungsgleiohung fiì. $F$ übergeht in

$$
r \frac{d^{2} F}{d r^{2}}+\frac{d F}{d r}-\frac{F}{r}-\frac{1}{h} \frac{d h}{d r}\left(r \frac{d F}{d r}-\frac{1}{n} F\right)+\left(3+\frac{1}{m}\right) \mu \omega^{2} h r^{2}=0 \quad .
$$

Drückt man die Veränderlichkeit von $h$ durch ein Potenzgesetz in $r$ mit beliebig gegebenem Exponenten $n$ aus:

so wird aus Gl. (7)

$$
h=c r^{n}
$$

$$
r \frac{d^{2} F}{d r^{2}}+(1-n) \frac{d F}{d r}-\left(1-\frac{n}{m}\right) \frac{F}{r}+\left(3+\frac{1}{m}\right) \mu \omega^{2} c r^{n}+2=0 .
$$

mit der allgemeinen Lösung:

wobei $a$ sich bestimmt aus:

$$
F=a r^{n+3}+A r^{\alpha}+B r^{\beta} \text {. }
$$

$$
a=-\frac{\left(3+\frac{1}{m}\right) \mu w^{2} c}{\frac{n}{m}+3 n+8} .
$$

und $\alpha$ und $\beta$ die Wurzeln der Gleichung

$$
x^{2}-n x+\frac{n}{m}-1=0
$$

sind, während $A$ und $B$ die beiden Integrationskonstanten bedeuten, die den jeweiligen Randbedingungen anzupassen sind.

Durch Aneinanderreihen von mehreren Aesten, von denen jeder gut durch einen Ansatz nach $\Lambda$ rt von Gl. (8) dargestellt wird, läßt sich jede vorgegebene Veränderlichkeit der Dicke mit hin reichender Genauigkeit wiedergeben.

Soweit ist die Theorie der rotierenden Scheiben mit veränderlicher Dicke bekannt und an zahlreichen Beispielen sind numerische Rechnungen durchgeführt worden. Wir werden nun zeigen, daß, wir Schritt für Sohritt die gleichen Ueberlegungen bei der $\mathrm{Be}$ anspruchung symmetrisch belasteter Kreisplatten anwenden können und auch zu denselben Lösungen gefïhrt werden.

2. Symmetrisch belastete Kreisplatte mit veränderlicher Dicke. Wir denken uns die Platte mit verändellicher Dicke ähnlich wie die Scheibe so ausgebildet, da $B$ die Plattenmittelfläche vor der Formänderung eine Ebene ist und zwar eine Symmetrieebene für die Gestalt der Platte. Wie nnter 1, wollen wir uns aus der Kreisplaite ein Element durch benachbarte Radial- und Kreisschnitte senkrecht zur Mittellläche herauggeschnitten denken. Nach der Kirchhoffschen Plattentheorie sind die Spannungen in diesen Schnitten nach dem Geradliniengesetz verteilt. Wir sprechen hier gar nicht von den Spannungen, sondern gleich von den resultierenden Biegungsmomenten, bezogen auf die Langeneinheit in der Plattenmittelfläche. Die Biegungsmomente, zu denen die Spannungen in den Scbnittflächen des Plattenelementes Veranlassung geben, nennen wir $M_{r}$ bezw. $M_{t}$. Sie entsprechen den Spannungsresultanten $T_{r}$ und $T_{t}$ bei der Scheibe. Außer den Biegungsspannungen treten aber bei der Platte noch Schubspannungen auf. Aus Symmetriegrüinden sind in den Radialschnitten die Schubspannungen null; auch in den Tangentialschnitten verschwinden aus dem gleichen Grund die Sohubspannungen parallel zur Mittelfläche; dagegen sind in diesen Sehnitten die Sohubspannungen in Richtung senkrecht zur Mittelfläche von null verschieden und geben Veranlassung zu einer resultierenden Scherkraft $V$, die wir uns auch wieder auf die Längeneinheit in der Platten-

1) A. und L. Foppl, Drang und Zwang, München 1920, Bd. I, \$ 54. 
mittelfläche bezogen denken wollen. Das. Gleichgewicht des Plattenelementes gegen Verdrehen um eine in Richtung der Tangente an den Kreisschnitt $r$ verlaufende Gerade lantet dann:

$$
\frac{d M_{r}}{d r}=\frac{M_{t}-M_{r}}{r}-V \text {. . . . . . . . . . . . . ( (1'), }
$$

eine Gleichung, die ähnlich gebaut ist wie Gl. (1). Die resultierende Scherkraft $V$ folgt ans der Gleichgewichtsbedingung des Plattenelementes in vertikaler Richtung. Wird die Lastintensität mit $p$ bezeichnet, so ergibt dies

$$
\frac{d\left(V_{r}\right)}{d r}=p r .
$$

Die Größe $V$ läßt sich demnach aus der gegebenen Belastung $p$ leicht berechnen, so daB wir sie als Bekannte in Gl. $\left(1^{\prime}\right)$ ansehen wollen. Die rein statischen Bedingungen der Aufgabe sind damit erschöpft. Sie liefern eine Gleichung für die beiden Unbekannten $M_{r}$ und $\boldsymbol{M}_{\boldsymbol{t}}$.

Um die Gestaltsänderung der Platte zu kennzeichnen, genügt es, die Krümmung der ursprünglich obenen Plattenmittelfläche an jeder Stelle anzugeben. Da die Mittelfläche Achsensymmetrie besitzt, sind die Hauptkrïmmungen an jeder Stelle durch die Krümmung $x_{r}$ des Meridianschnittes und die Krïmmung $x_{t}$ senkrecht dazu bestimmt. Sie lassen sich beide mit Hilfe des Neigungswinkels $p$, den die Tangente an die Meridiankurve der Mittelfläche mit der Horizontalen bildet, ausdrücken. Es ist nämlich

$$
x_{r}=\frac{d \varphi}{d}, \quad x_{t}=\frac{\varphi}{r} .
$$

Die Formänderungsgrößen der Platte $x_{r}, x_{t}$ und $p$ einerseits und der Scheibe $\varepsilon_{r}$, $\varepsilon_{t}$ und $\varrho$ andererseits entsprechen sich gegenseitig.

Das Hookescbe Gesetz lautet hier

$$
\frac{d \varphi}{d r}=\frac{1}{E J}\left(M_{r}-\frac{1}{m} M_{t}\right), \quad \stackrel{\varphi}{r}=\frac{1}{E J}\left(M_{t}-\frac{1}{m} M_{r}\right),
$$

wobei das Trägheitsmoment $J$ sich ebenso wie die Biegungsmomento anf die Längeneinheit in der Mittellläche bezieht, so daß

$$
J=\frac{\boldsymbol{B}^{3}}{12}
$$

ist, wonn die Plattendicke im Gegensatz zur Scheibendicke $h$ mit $H$ bezeichnet wird.

Die Hookeschen Gleichungen nach den Momenten aufgelöst lauten:

$$
M_{r}=\frac{m^{2}}{m^{2}-1} E J\left(\frac{d \varphi}{d r}+\frac{1}{m} \frac{\varphi}{r}\right), \quad M_{i}=\frac{m^{2}}{m^{2}-1} E J\left(\frac{\varphi}{r}+\frac{1}{m} \frac{d \varphi}{d r}\right) \quad .
$$

Darin wird gewöhnlich

$$
\frac{m^{2}}{m^{2}-1} E J=N
$$

die "Plattensteifigkeit" genannt, die hier mit $f$ bezw. $H$ von $r$ abhängig ist. Das Trägheitsmoment $J$ spielt bier dieselbe Rolle wie die Dicke $h$ bei der Scheibe.

Wir können $M_{r}$ und $M_{t}$ nach den Gl. (3') in Gl. (1') einsetzen und erhalten eine Gleichung für $p$, die natürlich dieselbe Form haben muB wie Gl. (4), wenn man die sich entsprechenden GröBen einsetzt. Sie lautet:

$$
\frac{d^{2} \varphi}{d r^{2}}+\frac{1}{r} \frac{d \varphi}{d r}-\frac{\varphi}{r^{2}}+\frac{1}{J} \frac{d J}{d r}\left(\frac{d \varphi}{d r}+\frac{1}{m} \frac{\varphi}{r}\right)=-\frac{V}{J} \frac{m^{2}-1}{m^{2} E}
$$

Dabei ist zu beachten, daß die sich entsprechenden Größen $\varrho$ und $\Phi$ von verschiedener Dimension sind. Um auch darin Uebereinstimmung zu erzielen, maltipliziere man etwa Gl. (4') mit dem Radius $a$ der Platte bezw. Scheibe.

Machen wir von dem anderen Weg zur Lösung Gebrauch, den wir in 1 gezeigt haben, so ergibt die Elimination von $\varphi$ aus den Gl. $\left(3^{\prime}\right)$

$$
\cdot \frac{1}{J}\left(M_{r}-\frac{1}{m} M_{t}\right)=\frac{a}{d r}\left[\frac{r}{J}\left(M_{t}-\frac{1}{m} M_{r}\right)\right]
$$

Zusammen mit $\mathrm{Gl}$. $\left(1^{\prime}\right)$ bestimmt diese Gleichung die Momente $M_{\mathrm{r}}$ und $M_{i}$. Wir fiihren wieder eine Art Spannungsfunktion $F$ ein durch

$$
M_{r}=\frac{a F}{r}, \quad M_{t}=a \frac{d F}{d r}+V r .
$$


wodurch Gl. ( $1^{\prime}$ ) identisch befriedigt wird und Gl. $\left(5^{\prime}\right)$ die folgende Bedingungsgleichung für $F$ liefert:

$$
r \frac{d^{3} W^{\prime}}{d r^{2}}+\frac{d F}{d r}-\frac{F}{r}-\frac{1}{J} \frac{d J}{d r}\left(r \frac{d F}{d r}-\frac{1}{m} F\right)+\frac{r^{2}}{a} \frac{d V}{d r}+\frac{r}{a} V\left(2+\frac{1}{m}-\frac{r}{J} \frac{d J}{d r}\right)=0 .
$$

Wir müssen nnn nochmals auf die schon oben abgeleitete Gleichung, aus der sioh die Scherkraft $V$ bestimmt, zurückgreifen:

$$
\frac{d(V r)}{d r}=p r .
$$

Welche von den üblichen Belastungen $p$ auch gegeben sein mag und wie sich auch dementsprechend aus der letzten Beziehung $V$ berechnet, so labt sich stets Gl. $\left(7^{\prime}\right)$ anf die gleiche Weise lösen, wie wir es unten für den besonderen Fall der strengen Analogie mit der rotierenden Scheibe angeben werden, so daß sich demnach der allgemeinste Fall einer symmetrisoh belasteten Kreisplatte, die am Rand frei gelagert oder auch eingespannt ist, ohne Schwierigkeit erledigen läßt.

Um die vollkommene Analogie zwischen rotierender Scheibe und Kreisplatte zu Finde zu fübren, sind noch die folgenden "Aebnlichkeitsbedingıngen* zu erfüllen, wie ein Vergleich der Gl. (4) und (4') zeigt:

$$
J=a^{2} h, \quad a \frac{v}{J}=\mu \omega^{2} r .
$$

Der Faktor $a^{2}$ in der ersten Gleichung dient daza, die Dimensionen auf beiden Seiten der Gleichung richtig zu stellen. Die zweite Aehnlichkeitsbedingnng läßt sich durch Einführung der Belastung $p$ auch folgendermaßen sehreiben

Danit geht Gl. $\left(7^{\prime}\right)$ über in

$$
p=\frac{\mu \omega^{3} d\left(J r^{2}\right)}{a r d r} \text { oder } p=\mu \omega^{2} \frac{a}{r} \frac{d\left(h r^{2}\right)}{d r} .
$$

$$
r^{\frac{d^{2} F^{\prime}}{d r^{2}}+\frac{d F}{d r}-F}-\frac{1}{r}-\frac{d J}{d r}\left(r \frac{d F}{d r}-\frac{1}{m} F\right)+\left(3+\frac{1}{m}\right) \mu \omega^{2} r^{r^{2}} J=0
$$

Wegen $\frac{J}{a^{2}}=h$ ist diese Gleichung identisch mit Gl. (7). Wie im AnschluB an Gl. (7), läßt sich hier selbstverständlich ebenso die Lösung von Gl. (7") ohne Schwierigkeit angeben.

Die Analogie zwischen rotierender Scheibe und belasteter Kreisplatte ist demnach in allen Einzelbeiten durchfïhrbar und eine Lösung der einen Aufgabe liefert unmittelbar auch eine Lösung der anderen. Die sich entsprechenden Größen seien hier nochmals zusammengestellt, wobei durch Multiplikation bezw. Division mit dem konstanten Radius $a$ für Gleichheit der Dimensionen entsprechender Gröben gesorgt ist.

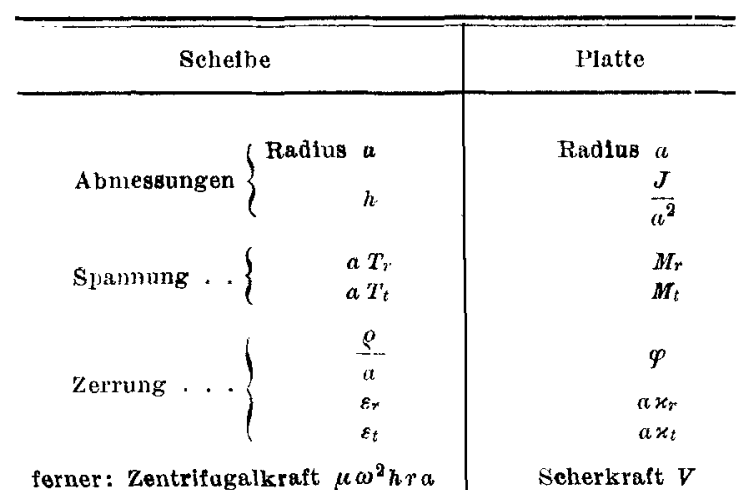

Aus dem letz'en Vergleichspuokt folgt die Bedingung für die Belastungsintensität $p$ der Platte:

$$
p=\mu \omega^{2} \frac{a}{r} \frac{d\left(h r^{2}\right)}{d r} .
$$

Damit ist der. Vergleich vollkommen durchgeführt. 
Für konstante Dicke $h=\frac{J}{a^{2}}=$ konst. folgt für die Belastnng:

$$
p=2 \mu \omega^{2} a h=\text { konst. }
$$

3. Beispiel. Bei rotierenden Scheiben gleicher Festigkeit $\left(\sigma_{v}=\sigma_{t}=\sigma=\right.$ konst.) wird für die Dicke $h$ folgende Abhängigkeit von $r$ gefunden '):

$$
h=h_{0} e^{-\frac{\mu\left(\theta^{2}\right)^{2}}{2} r^{2}} \text {. }
$$

Wenn sich diese Gestalt der Scheibe auch nicht genau nachahmen läßt, da erst für $r=\infty$ die Dicke za null würde, so liefert sie doch einen brauchbaren Anhaltspunkt fïr die Abnahme der Dicke mit $r$, und in der Tat stimmt die Form der bei der Lavalturbłne verwendeten Laufräder gut damit überein.

Wir wollen die Dicke der Kreisplatte und ihre Belastungsintensitat $p$ angeben, die der obigen Scheibe entspricht. Die Dicke $H$ bestimmt sich aus

$$
J=\frac{B^{3}}{12}=a^{2} h=a^{2} h_{0} e^{-\frac{\mu \omega^{2}}{2 J} r^{2}}
$$

oder, indem wir mit $H_{0}=\sqrt[3]{12 a^{2} h_{0}}$ die Dicke in der Mitte für $r=0$ bezeichnen:

$$
H=H_{0} e^{-\frac{\mu \omega^{9}}{6 \sigma} r^{2}} \text {. }
$$

Die Belastung folgt aus

$$
p=\mu \omega^{2} \frac{a}{r} \frac{d\left(h r^{2}\right)}{d r}=\mu \omega^{2} h a\left(2+\frac{r}{h} \frac{d h}{d r}\right), \quad \text { zu } \quad p=-2 \mu \omega^{2} h a-\frac{\mu^{2} \omega^{4}}{\sigma} a r^{2} h .
$$

Die Belastungsintensität $p$ hat demnach in der Mitte den Wert

$$
p_{0}=2 \mu \omega^{2} h a \text {. }
$$

Am Rand $r=a$ liegt die Platte trei auf, da hier $M_{r}=0$ ist, entsprechend $\sigma_{r}=0$ bei der Scheibe.

Im Anschluß an diese Aufgabe liegt es nahe, nach der Platte gleicher Festigkeit, elwa bel konstanter Belastung $p$, zu fragen. Diese Frage läbt sich nicht einfach beantworten, dagegen läßt sich die Bedingung

$$
\frac{M_{r}}{J}=\underset{J}{M_{t}}=C=\text { konst. }
$$

bei gleichmäßig verteilter Belastung einfach durchführen und liefert als Gesetz für die Abnahme der Dicke $B$ der Platte: $\quad C\left(H^{3}-H_{0}^{3}\right)=3 p r^{2}$.

Die obige Bedingang bedeutet einen überall gleicbmä日igen Anstieg der Spannungen von der Mittelfläche aus, so daß bei dem angegebenen Dickengesetz die Kantenspannungen an der Stelle der größten Dicke $H_{9}$ am größten ist. Durch einen noch etwas stärkeren Abfall der Dicke $H$ mit wachsendem $r$ kann man einer überall gleichen Kantenspannung nahe kommen. Auf weitere Einzelheiten in dieser Frage einzugehen läBt sich mit dem $\mathrm{Z}$ weck dieses Aufsatzes nicht vereinigen.

\section{Die Entwicklung der laminaren Geschwindigkeitsverteilung und ihre Bedeutung für Zähigkeitsmessungen. ${ }^{2}$ )}

(Mit cinem Anhang aber den Druekverlust turbulenter Strömung beim Eintritt in ein Rohr.)

\section{Von L. SCHILLER in Leipzig.}

$\mathrm{G}$ elegentlich einer experimentellen Untersuchung über den krilischen Wert der Reynoldsschen Zahl (reduzierten Geschwindigkeit) ${ }^{3}$ ) wurde der Widerstand der Laminarströmung bei höheren Rey nolds schen Zahlen beträchtlich größer gefunden, als dem Pois euille schen Gesetze entspricht; die Differenz wuohs mit zunehmender Geschwindigkeit. Als Grund dieser Erscheinung ergab sich, dab innerhalb der Mebstrecke nicht durchwegs die von Poisenille vorausgeselzte parabolische Geschwindigkeitsver-

\footnotetext{
1) Th. v. Kármán, Enzykl. d. matb. Wissensch, Bd. IV, Art. 27, Nr. $11 \mathrm{~b}, \mathbf{8 .} 361$.

2) Aus der Leipziger Habilitationsschrift des Verfassers.

3) L. Schiller. Experimentelle Untersuchungen zam Tarbulenzproblem. Dlese Zeitschr. 1, S. 436,
} 1921. Hinweise auf diese Arbelt werden im folgenden durch Sch. angegeben. 